\title{
Identification of putative biomarkers for the serodiagnosis of drug-resistant Mycobacterium tuberculosis
}

Lu Zhang ${ }^{1 *}$, Qingzhong Wang ${ }^{2}$, Wenjie Wang ${ }^{1}$, Yanyan Liu', Jie Wang ${ }^{3}$, Jun Yue ${ }^{3}$, Ying Xu' ${ }^{1}$ Wenxi Xu', ZhenLing Cui ${ }^{3}$, Xuelian Zhang ${ }^{1}$ and Honghai Wang ${ }^{1 *}$

\begin{abstract}
Background: Early diagnosis and treatment of Mycobacterium tuberculosis infection can prevent most deaths resulting from this pathogen; however, multidrug-resistant strains present serious threats to global tuberculosis control and prevention efforts. In this study, we identified antigens that could be used for the serodiagnosis of drug-resistant M. tuberculosis strains, using a proteomics-based analysis.

Results: Serum from patients infected with drug-resistant or drug-susceptible M. tuberculosis strains and healthy controls was subjected to two-dimensional gel electrophoresis using a western blot approach. This procedure identified nine immunoreactive proteins, which were subjected to MALDI-TOF-MS analysis. Six recombinant proteins, namely rRv2031c, rRv0444c, rRv2145c, rRv3692, rRv0859c, and rRv3040, were expressed and used to determine the immuno-reactivity of 100 serum samples. Antibody reactivity against rRv2031C, rRv3692, and rRv0444c was consistently observed. Among them, the best sensitivity and specificity of rRv3692 were $37 \%$ and 95\% respectively. Furthermore, when rRv2031c and rRv3692 or rRv2031c, rRv3692, and rRv0444c were combined in 2:1 or equal amounts, the assay sensitivity and specificity were improved to $56.7 \%$ and $100 \%$ respectively.
\end{abstract}

Conclusions: These results suggest that Rv2031c, Rv3692, and Rv0444c are possible candidate biomarkers for effective use in the serodiagnosis of drug-resistant tuberculosis infections, and a combined formula of these antigens should be considered when designing a subunit assay kit.

Keywords: Immunoproteomics, Mycobacterium tuberculosis, Drug-resistance, Serodiagnosis

\section{Background}

More than one-third of the world's population is infected with tubercle bacilli. Poor adherence to tuberculosis (TB) control programs, the subsequent emergence of drug-resistant strains and the widespread prevalence of HIV have contributed to the resurgence of this disease [1]. Multidrug-resistant tuberculosis (MDRTB) and extensively drug-resistant tuberculosis present serious threats to global TB control efforts. The World Health Organization estimated that the number of new MDR-TB cases in 2004 was 425,000 , and $60 \%$ of these

\footnotetext{
* Correspondence: zhanglu407@fudan.edu.cn; hhwang@fudan.edu.cn 'State Key Laboratory of Genetic Engineering, Institute of Genetics, School of Life Science, Fudan University, 220 Handan Road, 200433 Shanghai, Peoples Republic of China

Full list of author information is available at the end of the article
}

cases were located in China, India, and the Russian Federation $[2,3]$.

Most TB-associated deaths are preventable with early diagnosis and treatment [4], and it has been suggested that a quick and accurate tool for early TB diagnosis could save up to 625, 000 lives each year [5]. Currently, optimal diagnostics for TB are a demanding task and new procedures are urgently needed. In endemic areas, sputum-smear microscopy is often the only available and affordable diagnostic test but is only $50-70 \%$ sensitive. The diagnostic gold standard is considered to be culturing Mycobacterium tuberculosis, as it is sensitive and specific in cases of smear-positive TB. However, results usually take two to four weeks and this test is not routinely used in countries with high prevalence of TB. Recently developed PCR-based tests or interferon- 
release assays, such as QuantiFERON ${ }^{\circledR}$ TB-Gold, are expensive and require a certain level of expertise [4].

Antibody detection is one of the most common approaches used in the diagnosis of infectious diseases. Antibody production is typically the result of antigen exposure from an infection, and dominant antigens are the primary target for diagnostic and immunoprophylactic therapies. Detection of $M$. tuberculosis antigens released during the active disease stage has been considered a specific and ideal approach in the serodiagnosis of TB because these antigens represent an early disease state [6]. More specifically, antigens including antigen 5 (38 kDa antigen, Rv0934), $27 \mathrm{kDa}$ antigen (MPT51, Rv3803c), $30 \mathrm{kDa}$ antigen (Ag85B, MPT59, Rv1886c), P32 (Rv3804c), $88 \mathrm{kDa}$ antigen (MTB81, Rv1837c), antigen 60 (A60), cord factor, Kp90 and LAM could potentially be used in the serodiagnosis of TB [7]. To date, there are no commercially available serodiagnostic tests for TB. In particular, there is no routine laboratory test for drug-resistant TB with sufficient sensitivity and specificity.

Proteomics is a powerful tool for studying the protein composition of complex biological samples. Protein expression profiles of $M$. tuberculosis under various growth conditions, genetic backgrounds and geographic distribution have been investigated [8-15]. The application of immunoproteomics, combining 2-DE with immunoblotting to find protein candidates for serodiagnostics, is a powerful tool [16-18]. Using this approach, Bassey et al. found some heterogeneity in the responses to $M$. tuberculosis antigens with different patient sera [19]. Karen et al. identified 12 immunogenic proteins from actively replicating $\mathrm{H} 37 \mathrm{Rv}$ culture filtrates [20]. However, until recently, there was little information regarding the immunogenicity profiles associated with drug-resistant $M$. tuberculosis strains.

Therefore, the main objective of this study was to evaluate differences between antigen expression and the respective immunoreactivity profiles of drug-susceptible and drug-resistant $M$. tuberculosis strains using an immunoproteomic approach. Identified proteins were subsequently cloned, expressed and purified, and their subcellular location was determined. Antibody reactivity against these proteins was detected by ELISA to evaluate the potential of these antigens to be used as biomarkers for early identification of MDR-TB.

\section{Results and discussion}

\section{M. tuberculosis 2-DE profile of whole cell proteins}

Two-dimensional gel electrophoresis analysis was performed on five MDR clinical isolates and H37Rv, which is a strain that is sensitive to all four first-line TB antibiotics. Representative 2-DE gels of these strains are presented in Figure 1. In the $\mathrm{pH}$ range of 4-7, approximately 1000 silver-stained protein spots were reproducibly detected in all strains using the PDQuest version 6.0 software. Three or more gels for each strain were analyzed and compared during the course of this analysis. Only gels with similar normalized volumes for each sample analyzed were selected for western blot analysis.

\section{Identification of immunoreactive proteins}

Proteins from MDR and antibiotic-susceptible strains were examined by western blot for immunoreactivity to serum from patients infected with drug-resistant $M$. tuberculosis. Pooled serums from patients infected with antibiotic-susceptible $M$. tuberculosis were used as negative controls. Figure 1B, C reflect representative antigenic profiles using serum collected from individuals infected with drug-resistant $M$. tuberculosis and drugsusceptible $M$. tuberculosis, respectively. Immunoreactive protein spots selected for further analysis were present on blots probed with patient serum but not visible on blots probed with negative controls. Protein mass fingerprinting of trypsin digest fragments was performed on these proteins, and the results are summarized in Table 1 (including data sourced from Additional files 1, 2, 3, 4, 5, 6, 7 \&8). Protein characterization was conducted using MALDI-TOF-MS and MALDI-TOF-MS/MS analysis, and the data were compared with the MASCOT database for sequence matches. Identification of proteins was based on the probability scores for the matches, molecular mass, pI, number of peptide matches and the percentage of the total translated ORF sequence covered by the peptides.

Pooled sera from patients diagnosed with drug-resistant $M$. tuberculosis infection were used to identify immunodominant antigens. The identification of $M$. tuberculosis proteins by human sera suggested that these antigens were expressed in vivo and were capable of eliciting humoral responses. These antigens could therefore be considered attractive immunodiagnostic candidates. A total of nine immunoreactive protein spots (Figures 1,2) were identified and are reported in Table 1. Among them, the M. tuberculosis proteins Rv0639, Rv3028c, Rv3040, Rv2145c, Rv0859c, Rv3692 and Rv0444c were only present when MDR strains were probed with serum from patients infected with MDR $M$. tuberculosis. These proteins were not observed on membranes probed with negative control serum. Rv1310 was identified on all membranes examined. Rv2031c was only present on membranes blotted with drug-susceptible strains and serum from patients infected by antibiotic-sensitive clinical strains (Figure 3).

The nine unique MDR proteins were divided into four functional groups. The first group encompassed metabolic pathway-associated proteins, including carbohydrate 


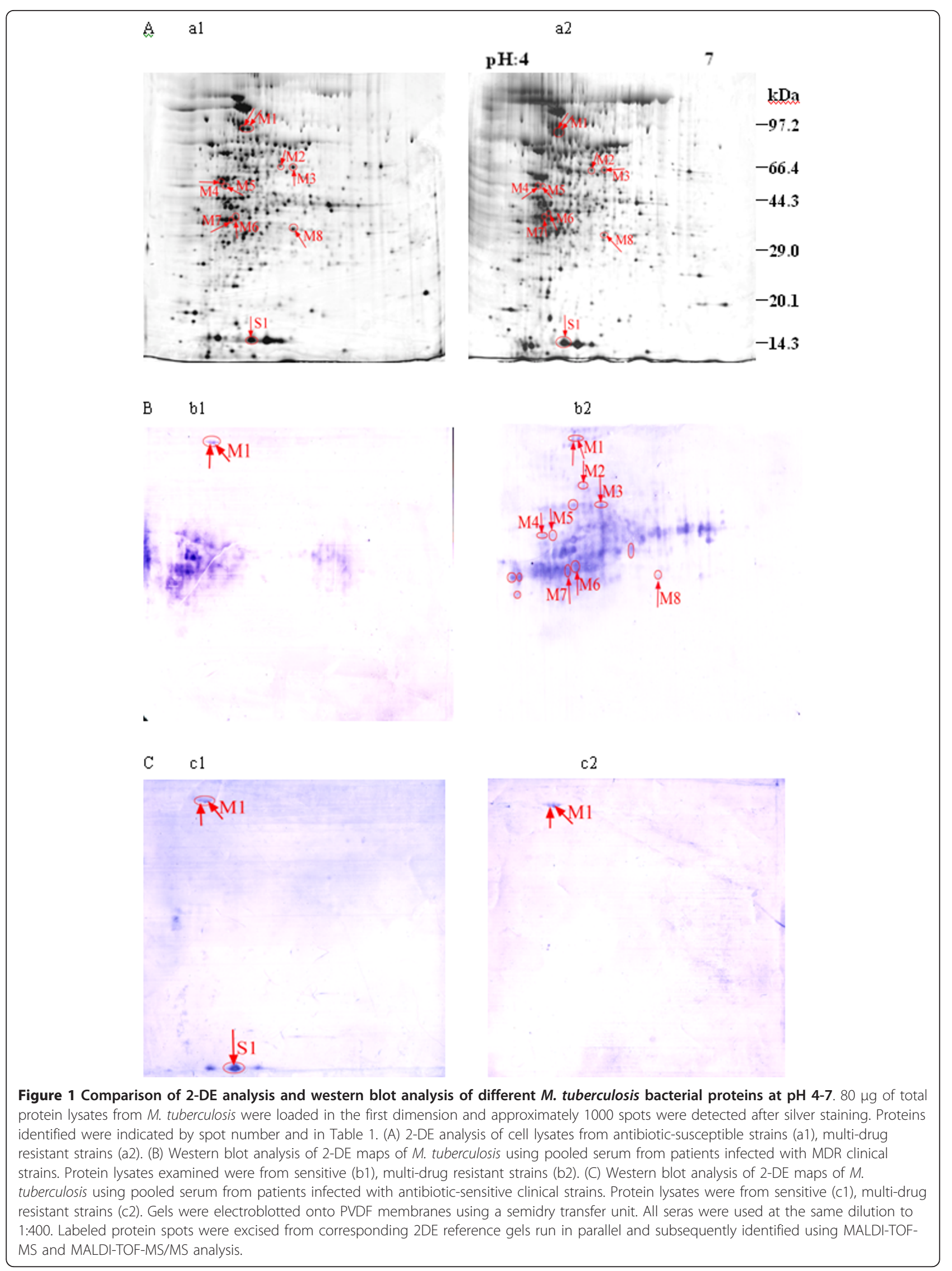


Table 1 Immunoreactive protein spots identified by MALDI-TOF-MS

\begin{tabular}{|c|c|c|c|c|c|c|c|c|c|c|}
\hline \multirow{2}{*}{$\begin{array}{l}\text { Spot } \\
\text { No }^{\mathrm{a}} \text {. }\end{array}$} & \multirow{2}{*}{$\begin{array}{c}\text { NCBI } \\
\text { accession } \\
\text { no. }^{\text {b }}\end{array}$} & \multirow{2}{*}{$\begin{array}{l}\text { Sanger } \\
\text { I.D. }\end{array}$} & \multirow[t]{2}{*}{ Protein identification } & \multicolumn{2}{|c|}{ MW/PI } & \multirow{2}{*}{$\begin{array}{l}\text { Mascot } \\
\text { score }^{c}\end{array}$} & \multirow{2}{*}{$\begin{array}{l}\text { GRAVY } \\
\text { score }^{d}\end{array}$} & \multicolumn{3}{|c|}{ 2D immunoreactivity } \\
\hline & & & & Theoretical & Experimental & & & $\begin{array}{c}\text { Sensitive } \\
M . \\
\text { tuberculosis }\end{array}$ & $\begin{array}{c}\mathrm{RIF}^{\mathrm{r}} M . \\
\text { tuberculosis }\end{array}$ & $\begin{array}{l}\text { MDR } M \text {. } \\
\text { tuberculosis }\end{array}$ \\
\hline M1 & gi|1322434 & Rv1310 & $\begin{array}{c}\text { ATP synthase beta chain } \\
\text { (atpD) }\end{array}$ & $\begin{array}{c}53093.32 / \\
4.76\end{array}$ & $53061.2 / 4.86$ & 433,57 & -0.168 & S-sera ${ }^{e}$ & All sera & All sera \\
\hline M2 & gi|2916918 & Rv0859c & Acyl-CoA thiolase (fadA) & $\begin{array}{l}42413.60 / \\
5.14\end{array}$ & $42387.8 / 5.2$ & 181,32 & 0.035 & R-sera & - & - \\
\hline M3 & $\begin{array}{c}\text { gi| } \\
15610828 \\
\end{array}$ & Rv3692 & $\begin{array}{l}\text { Methanol dehydrogenase } \\
\text { regulatory protein(moxR2) }\end{array}$ & $\begin{array}{c}37903.47 / \\
5.51\end{array}$ & $37904.3 / 5.43$ & 192,20 & -0.002 & - & R-sera & - \\
\hline M4 & $\begin{array}{c}\text { gi| } \\
15610165\end{array}$ & Rv3028c & $\begin{array}{c}\text { electron transfer } \\
\text { flavoprotein (alpha } \\
\text { subunit) (fixB, etfA) }\end{array}$ & $\begin{array}{c}31690.02 / \\
4.61\end{array}$ & $31699.8 / 4.47$ & 746,68 & 0.358 & - & - & R-sera \\
\hline M5 & gi|2791638 & Rv3040 & $\begin{array}{l}\text { Conserved hypothetical } \\
\text { protein }\end{array}$ & $\begin{array}{l}31484.26 / \\
4.50\end{array}$ & $31465.7 / 4.65$ & 98,6 & -0.297 & - & - & R-sera \\
\hline M6 & gi|2104333 & $R v 2145 c$ & antigen 84(wag31) & $\begin{array}{l}28276.23 / \\
4.75\end{array}$ & $28260.1 / 4.80$ & 133,49 & -0.726 & - & - & R-sera \\
\hline M7 & $\begin{array}{l}\text { gi| } \\
15607779\end{array}$ & Rv0639 & $\begin{array}{c}\text { Transcription } \\
\text { antitermination protein } \\
\text { (nusG) }\end{array}$ & $\begin{array}{l}25413.54 / \\
4.59\end{array}$ & 25431.0/4.70 & 175,23 & -0.180 & - & - & R-sera \\
\hline M8 & $\begin{array}{c}\text { gi| } \\
15607585\end{array}$ & Rv0444c & $\begin{array}{c}\text { Conserved hypothetical } \\
\text { protein }\end{array}$ & $\begin{array}{c}23882.15 / \\
5.82\end{array}$ & 23883.0/5.81 & 75,13 & 0.062 & - & R-sera & - \\
\hline S1 & gi|2896768 & Rv2031C & $\begin{array}{l}\text { Heat Shock Protein hspX } \\
\text { (14-kDa antigen) }\end{array}$ & $\begin{array}{c}16226.30 / \\
4.90\end{array}$ & 16217.2/5.0 & 209,35 & -0.52 & - & S-sera & - \\
\hline
\end{tabular}

a) Spot numbers refer to the numbers on the 2-DE gels showed in Figure $1 \mathrm{~A}-\mathrm{C}$.

b) Accession no. obtained from the MASCOT results of MALDI-TOF/TOF from the NCBInr database http://www.ncbi.nlm.nih.gov.

c) MASCOT score and percent sequence coverage of all tryptic peptides identified by MALDI-TOF-MS/MS. MASCOT protein scores (based on combined MS and MS/MS spectra (Additional files 1, 2, 3, 4, 5, 6, 7 and 8) greater than 72 were considered statistically significant ( $p \leq 0.05$ ).

d) Grand average of hydropathy.

e) Anti-sera from TB patients infected with antibiotic-sensitive M. tuberculosis.

f) Anti-sera from TB patients infected with drug-resistant $M$. tuberculosis
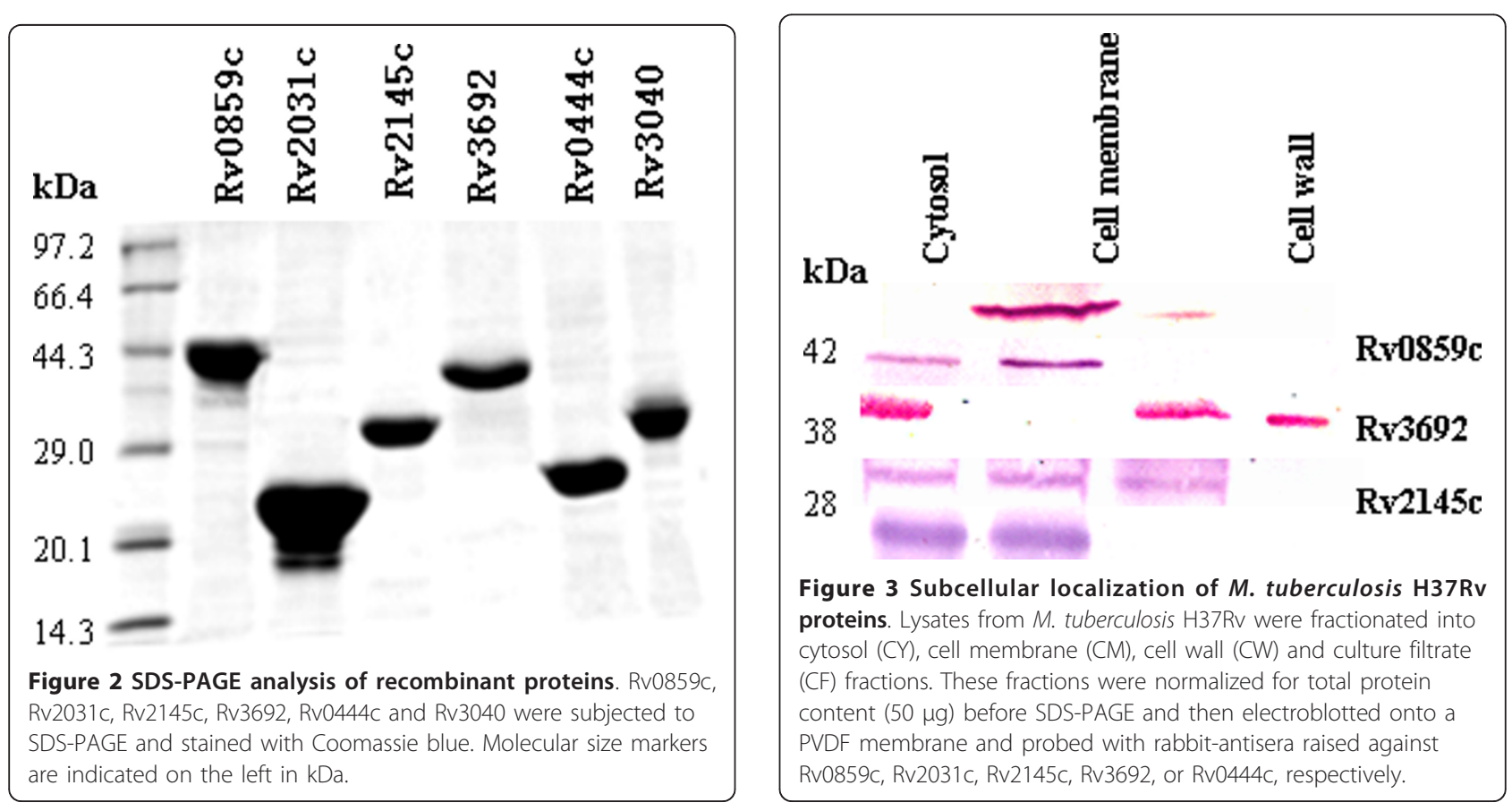
metabolism, lipid metabolism, respiratory chain, oxidation-phosphorylation and biotransformation. The functions of these proteins have been well characterized in $M$. tuberculosis, and included ATP synthase beta chain (EC 3.6.3.14) (AtpD), electron transfer flavoprotein (alpha subunit) (FixB, EtfA), acyl-CoA thiolase (EC 2.3.1) (FadA), and hydrodipicolinate reductase (EC 1.3.1.26) (DapB). These proteins represented the major protein spots on the gel. The second group included regulatory proteins whose functions have been biochemically defined, for example: transcription antitermination protein (NusG), methanol dehydrogenase regulatory protein (MoxR2) and Rv0444c, which is the SigK (RskA) regulator of certain M. tuberculosis complex members [21,22]. The third group comprised the $16 \mathrm{kDa}$ virulence factor antigen HspX, which is specific to the M. tuberculosis complex and is essential to bacilli survival, particularly during latency. This antigen has been used to detect antibody isotypes in sera of TB patients singly or in combination with other antigens [23]. Using the recombinant $38 \mathrm{kDa}$ and $16 \mathrm{kDa}$ antigens for immunodiagnosis provided sensitivities of $59 \%$ (culture positive) and $54 \%$ (culture negative) and specificity of $98 \%$ in patients with pulmonary TB. A recent study suggested that antibody responses to the $16-\mathrm{kDa}$ antigen may be important for the detection of latent TB infections [24,25]. Wag31, whose synonym is Ag84, made up the fourth group of proteins: those associated with cell wall and cell processes. The remaining protein, Rv3040c, is of unknown function. Undefined spots were considered to be either protein mixtures necessitating further separation or proteins present at concentrations below the detection limits of the assay (less than $1 \mathrm{ng}$ ).

\section{Recombinant protein expression and subcellular characterization}

Ten recombinant His-tagged $\mathrm{H} 37 \mathrm{Rv}$ proteins were cloned and expressed in this study. Six proteins were expressed at high levels both in soluble (rRv2031c, rRv0444c, rRv2145c, rRv3692) and insoluble (inclusion body) aggregates (rRv0859c, rRv3040). As shown in Figure 2, the purified His-tag fusion proteins moved as single bands on the sodium dodecyl sulfate polyacrylamide gel electrophoresis (SDS-PAGE) gels.

Initially, protein hydrophobicity was predicted by calculating the grand average of hydropathy scores using the ProtParam program http://www.expasy.org/tools/ protparam.html. A score of $>0.4$ indicated a hydrophobic protein likely to be membrane-associated [26]. Using these criteria, all of the identified spots from 2-DE were characterized as hydrophobic (Table 1). The subcellular location of five recombinant proteins was also investigated by western blot analysis using rabbit-antisera raised to the respective proteins. Four different subcellular fractions (culture filtrate, cytosol, cell membrane and cell wall) were analyzed for immunoreactivity. The fidelity of the fractions was confirmed as described previously [27]. Except for Rv2145c, which was present in culture filtrate and cell wall fractions, all proteins were located in the cell membrane of M. tuberculosis H37Rv. Several proteins were found in other locations as well as the cell membrane: Rv0859c was present in both cell wall and cell membrane fractions, Rv3692 was identified in the cytosol and cell membrane fractions, Rv0444c was present in the cytosol, cell wall and cell membrane fractions, and Rv2031c was present in the cytosol and cell membrane fractions. These data indicate that most immunogenic proteins detected using the immunoproteomic assay were located in the outer membrane fractions, suggesting that these proteins could function as candidate detection antigens.

\section{Evaluation of the serodiagnostic potential of the recombinant proteins}

All recombinant proteins were tested for their potential value in the serodiagnosis of TB by ELISA. Serum from 60 patients infected with MDR M. tuberculosis and 20 patients infected with antibiotic-susceptible $M$. tuberculosis was used in the analyses. Only three proteins, rRv2031c, rRv3692, and rRv0444c, were consistently immunoreactive to patient serum and were subjected to further evaluation under various combination strategies, such as rRv2031c and $\operatorname{rRv} 3692$ with a ratio of $1: 1,1: 2$, 1:3, 2:1, 3:1, 3:2, etc. Figure 4 shows the IgG seroreactivity to partial combinations of recombinant proteins at equal or mixed ratios. Combinations of rRv2031c and rRv3692 or rRv2031c, rRv3692, and rRv0444c provided
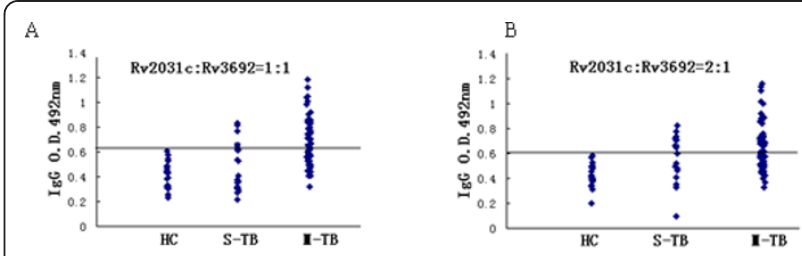

C
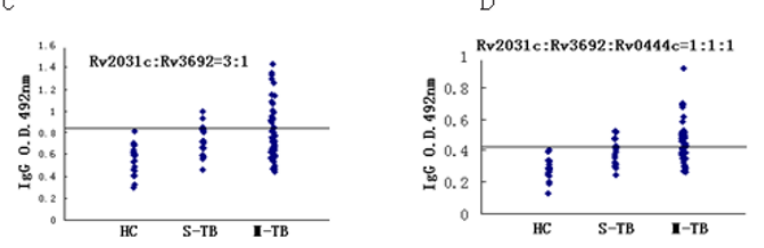

Figure 4 TB serodiagnose for clinical samples applied with different proteins. HC, healthy Mycobacterium bovis BCGvaccinated controls; S-TB, pulmonary tuberculosis patients infected by antibiotic-sensitive $M$. tuberculosis strains; M-TB, pulmonary tuberculosis patients infected by multi-drug resistant $M$. tuberculosis strains.Serum reactivity to (A) Rv2031c and Rv3692 at a 1:1 ratio, (B) Rv2031C and Rv3692 at a 2:1 ratio (C) Rv2031C and Rv3692 at a 3:1 ratio and (D) Rv2031c, Rv3692, and Rv0444c at a 1:1:1 ratio. 
significant levels of seroreactivity when probed with serum taken from drug-resistant $M$. tuberculosis infections, compared with the serum collected from healthy controls. An absorbance of 2 SD (standard deviation) over the mean OD of the healthy controls was considered as the cut-off criterion for seropositivity. Under these conditions, a combination of rRv2031c and rRv3692 at a 2:1 ratio, and the combination of rRv2031c, rRv3692, and rRv0444c with a ratio of $1: 1: 1$, gave a sensitivity of $56.7 \%$ and a specificity of $100 \%$, respectively (Table 2 and Figure 4). This indicated that antigens in combination could be potentially used in the serodiagnosis of drug-resistant TB. The use of a single protein in the detection assay showed lower sensitivity and specificity values than combinations of recombinant proteins, further demonstrating the value of multi-component detection formulations. Because the antibody responses to $M$. tuberculosis infections are heterogeneous, the desired sensitivity and specificity in serodiagnostic tests may be achieved by a combination of several proteins, or the fusion of several epitopes into a polyprotein.

Biomarkers of infection disease can indicate normal or pathogenic processes, or pharmacological responses to therapeutic intervention. So biomarkers provide prognostic information for individual patients or cohorts in clinical trials, either about future health status. The need for biomarkers in tuberculosis is most crucial in three areas: to indicate reactivation risk and predict treatment success for patients with latent M. tuberculosis infection; to predict durable (non-relapsing) treatment success for patients with active disease; and to indicate protection from tuberculosis by new vaccines

Table 2 IgG seroreactivity to recombinant $M$. tuberculosis proteins

\begin{tabular}{|c|c|c|c|c|}
\hline \multirow{2}{*}{\multicolumn{2}{|c|}{$\begin{array}{l}\text { Recombination } \\
\text { proteins }\end{array}$}} & \multirow{2}{*}{$\begin{array}{l}\text { MDR }^{\mathrm{a}} \\
\text { Total/Pos(\%) }\end{array}$} & \multirow{2}{*}{$\begin{array}{l}\text { Susceptible }^{\mathbf{b}} \\
\text { Total/Pos(\%) }\end{array}$} & \multirow{2}{*}{$\begin{array}{l}\text { Healthy controls } \\
\text { Total/Pos(\%) }\end{array}$} \\
\hline & & & & \\
\hline \multicolumn{2}{|l|}{ Rv0859c } & $60 / 14(23.3)$ & $20 / 1(5)$ & $20 / 0(0)$ \\
\hline \multicolumn{2}{|l|}{ Rv2031c } & $60 / 20(33.3)$ & 20/2(10) & $20 / 0(0)$ \\
\hline \multicolumn{2}{|l|}{ Rv2145c } & $60 / 13(21.7)$ & $20 / 0(0)$ & $20 / 0(0)$ \\
\hline \multicolumn{2}{|l|}{ Rv3692 } & $60 / 22(37)$ & 20/0(0) & $20 / 1(5)$ \\
\hline \multicolumn{2}{|l|}{ Rv0444c } & $60 / 15(25)$ & $20 / 3(15)$ & $20 / 0(0)$ \\
\hline \multicolumn{2}{|l|}{ Rv3040 } & $60 / 7(11.7)$ & 20/2(10) & 20/3(15) \\
\hline Rv2031c: & 1:1 & 60/33(55) & $20 / 5(25)$ & $20 / 0(0)$ \\
\hline \multirow[t]{2}{*}{ Rv3692 } & $2: 1$ & $60 / 34(56.7)$ & 20/10(50) & $20 / 0(0)$ \\
\hline & $3: 1$ & $60 / 21(35)$ & 20/7(35) & $20 / 1(5)$ \\
\hline \multicolumn{2}{|c|}{ Rv2031c:Rv3692: } & $60 / 34(56.7)$ & $20 / 9(45)$ & 20/0(0) \\
\hline
\end{tabular}

a) Serum from multi drug-resistant $M$. tuberculosis infected patients.

b) Serum from antibiotic-susceptible $M$. tuberculosis infected patients. for people other than those with active disease $[18,28]$. Peripheral blood is the most widely used source in clinical practice, although biomarkers can be studied in any tissue or body fluid(including urine, saliva, sputum and breath), Genes, transcripts, proteins, lipids and metabolites can all be measured in blood for biomarker studies [29].

Although this study identified several interesting immunoreactive $M$. tuberculosis proteins, not all could be used for the serological diagnosis of drug-resistant TB. To date we have analyzed immunogenic whole cell proteins that were resolved within the pI range of 4-7. The antigens identified so far largely depended on the combination of the source of the antigens, the immunoreactive immunoglobulin class (IgG or IgM) identified, stage of the diseases and ethnicity of the infected population. The conditions of the immunoproteomic approach (sample denaturation, loss of some proteins under 2-DE conditions) may have impaired the identification of additional antigens. An immunocapture procedure in which antigens could be enriched from subcellular fractions based on their reactivity with immobilized serum from patients infected by MDR could lead to the identification of novel candidate antigens. This kind of approach has already been successfully applied for other diseases [30,31]. It would be of interest to complete the database of potential antigen candidates using non-denaturing and complementary procedures.

\section{Conclusions}

Three candidate antigens, Rv2031c, Rv3692, and Rv0444c, identified in this study appeared to have greater antigenic activity. The antigens were therefore tested in combination using ELISA assays to evaluate the use of these proteins for the serodiagnosis of MDR M. tuberculosis infections. The results demonstrated that these antigens, when used in combination, were more effective than when used singly, suggesting that combinations of antigens may yield the desired level of sensitivity without affecting specificity. The validation phase of a diagnostic kit using a combination of rRv2031c and rRv3692 against a large panel of sera from patients infected with drug-resistant $M$. tuberculosis and healthy controls is also in progress. The aim of this work is to supply a commercial serodiagnostic test for drug-resistant TB with sufficient sensitivity and specificity.

To our knowledge, this is the first report that has identified antigen candidates for identifying drug-resistant TB using immunoproteomic analysis. Three candidate protein antigens in combination functioned effectively in the identification of immunoreactive antibodies from patients infected with MDR strains. While 
the cross-reactivity of the his tag and cleaved purified his-tag free antibody, and cross activity with MTM, are worth to further work to demonstrate.

\section{Methods}

Bacterial strains and culture conditions

M. tuberculosis strain TMC 102 [H37Rv] (ATCC number 27294) was provided by the Chinese Institute for the Control of Pharmaceutical and Biologic Products. Five clinical MDR M. tuberculosis isolates from different patients were obtained from the Shanghai Pulmonary Hospital. Their antibiotic susceptibility patterns were determined using the Bactec 960 instrument (Becton Dickinson Microbiology Systems, Sparks, MD, USA). The minimal inhibitory concentrations were determined using a 7H9 solid medium proportional method [32]. Strains were initially identified biochemically and then confirmed by $16 \mathrm{~S}$ rDNA gene sequencing.

\section{Human sera}

All patient serum samples were obtained from the Shanghai Pulmonary Hospital, China.

Blood samples were drawn from TB patients shortly after diagnosis (culture and/or acid-fast bacillus smear positive and chest $\mathrm{X}$-ray positive). All donors were between the ages of 18 and 65 and provided informed consent.

Serum was collected from patients infected with drugresistant $M$. tuberculosis $(n=20)$ or from patients infected with drug-susceptible M. tuberculosis $(n=20)$, then randomly divided into four different pools. Each pool included five serum samples and was used for immunoproteomic analysis. Serum from patients infected with drug-resistant M. tuberculosis $(\mathrm{n}=60)$ and drug-susceptible M. tuberculosis $(n=20)$ was collected for ELISA analysis of IgG reactivity to recombinant antigens. Serum from healthy volunteers was used as a negative control $(n=20)$. Serum reactivity to recombinant $M$. tuberculosis antigens were assessed by ELISA as described previously [20].

\section{Isolation of $M$. tuberculosis proteins}

Whole cell extracts were harvested from $M$. tuberculosis during early mid-log growth phase, as described previously [12]. Approximately $10^{9}$ bacteria were sonicated in the presence of proteinase inhibitors and then treated with $8 \mathrm{M}$ urea, $2 \mathrm{M}$ thiourea, $70 \mathrm{mM}$ dithiothreitol, $0.5 \%$ Biolyte (pH 4-7), $400 \mu \mathrm{g} / \mathrm{mL} n$-octyl-D-glucopyranoside, and $4 \%$ CHAPS to completely denature and reduce proteins. Supernatants were then collected after centrifugation at $10000 \times g$ for $15 \mathrm{~min}$. Three subcellular fractions (cell wall, membrane, and cytosolic fractions) of $M$. tuberculosis cell lysates were obtained by differential centrifugation as described previously [12].
Preparation of culture filtrate protein was also performed as described previously [33].

\section{Two-dimensional gel electrophoresis}

Proteins were identified by excision and in-gel trypsinization of respective spots from silver-stained gels as described previously [10]. The analyses were performed using a 4700 MALDI-TOF/TOF Proteomics Analyzer (Applied Biosystems, Foster City, CA, USA) equipped with a $355 \mathrm{~nm}$ Nd:YAG laser. Combined MS and MS/ MS spectra were submitted to MASCOT version 2.1 (Matrix Science, London, UK) using GPS Explorer software version 3.6 (Applied Biosystems). MASCOT protein scores (based on combined MS and MS/MS spectra) greater than 72 were considered statistically significant ( $\mathrm{p} \leq 0.05)$. The individual MS/MS spectrum with statistically significant (confidence interval > 95\%) best ion score (based on MS/MS spectra) were accepted. The number of identified peptides, sequence coverage and number of MS/MS identified peptides were considered during protein identification. To eliminate the redundancy of proteins that appeared in the database under different names and accession numbers, the single-protein member belonging to the species Mycobacterium with the highest protein score (top rank) was singled out from the multi-protein family [34]. Isoelectric focusing (IEF) was performed using an IPGphor IEF system (Bio-Rad, Hercules, CA, USA), and 2-DE was performed according to the manufacturer's instructions. Briefly, a 17 cm IPG strip pH 4-7 was rehydrated overnight in rehydration solution (7 M urea, $2 \mathrm{M}$ thiourea, 1\% ASB-14, 0.5\% Triton X-100, 0.5\% 3-10 carrier ampholytes, $55 \mathrm{mM}$ dithiothreitol, $0.002 \%$ bromophenol blue) containing $80 \mu \mathrm{g}$ of proteins in a total volume of $360 \mu \mathrm{L}$. Focusing was conducted by stepwise voltage increases as follows: $500 \mathrm{~V}$ for 2 hours, $1000 \mathrm{~V}$ for 2.5 hours, $8000 \mathrm{~V}$ for 5 hours and $8000 \mathrm{~V}$ until the total volt-hours reached $80 \mathrm{kVh}$. Following IEF separation, strips were equilibrated twice (14 min each time) in 8 $\mathrm{mL}$ of SDS equilibration buffer $(150 \mathrm{mM}$ Tris- $\mathrm{Cl}, \mathrm{pH}$ 8.8; $6 \mathrm{M}$ urea, $20 \%$ glycerol, $2 \%$ SDS, $0.002 \%$ bromophenol blue) containing $100 \mathrm{mg}$ dithiothreitol and $250 \mathrm{mg}$ iodoacetamide. IPG strips were then placed over a $12.5 \%$ vertical polyacrylamide gel $(0.75 \mathrm{~mm}$ thick, $18 \mathrm{~cm}$ wide, and $24 \mathrm{~cm}$ long), and electrophoresis was carried out at $30 \mathrm{~mA}$ until the bromophenol blue had run to the bottom of the gel. Second-dimension PAGE gels were run in duplicate, with the first gel used for immunoblotting and the second silver stained to serve as a 2$D$ reference map [35]. Gels were scanned using Molecular Image Fx (Bio-Rad) and analyzed with PDQuest version 6.0 software (Bio-Rad). For each sample, IPG gels were electrophoresed in triplicate on separate occasions. The gel images were normalized according to the total 
quantity in the analysis set as Griffin TJ [36]. Relative comparison of intensity abundance of differential protein spots was performed with Student's t-test. Any difference with a P-value $\leq 0.05$ was considered statistically significant.

\section{2-DE western blotting}

Proteins subjected to 2-DE gel electrophoresis were transferred onto $0.45 \mathrm{~mm}$ Immobilon-P PVDF membranes (Millipore, Billerica, MA, USA) using a semi-dry electro-blotter (Bio-Rad) with Towbin transfer buffer, at a constant current of $1 \mathrm{~mA} / \mathrm{cm}^{2}$ for $80 \mathrm{~min}$. PVDF membranes were incubated overnight in PBST $(9 \mathrm{mM}$ sodium phosphate, $0.15 \mathrm{M} \mathrm{NaCl}$, and $0.05 \% \mathrm{v} / \mathrm{v}$ Tween 20) containing $5 \%(\mathrm{w} / \mathrm{v})$ skim milk powder at $4{ }^{\circ} \mathrm{C}$ with constant rotation. Following three 10-min washes with PBST, blots were incubated for 1 hour at $37^{\circ} \mathrm{C}$ with human sera diluted 1:400 in PBST containing $2 \%(\mathrm{w} / \mathrm{v})$ skim milk powder. Human sera from uninfected volunteers and patients infected with drug-susceptible $M$. tuberculosis were used as negative controls. After washing with PBST, blots were incubated with biotinylated anti-human IgG (Vector Laboratories, Burlingame, CA, USA) at a dilution of 1:20, 000 for 1 hour at room temperature. Before and after the addition of the secondary antibody, membranes were washed four times for 15 $\mathrm{min}$ in PBST. Membranes were then washed with 50 $\mathrm{mM}$ Tris- $\mathrm{HCl}$ buffer ( $\mathrm{pH}$ 7.4) and developed with streptavidin-conjugated alkaline phosphatase (Vector Laboratories). Membranes were then incubated with nitro blue tetrazolium/5-Bromo-4-chloro-3-indolyl phosphate detection substrate. Immunoblotting experiments were conducted in triplicate and no variation was observed between results.

\section{Tryptic digests and MALDI-TOF-MS}

Proteins were identified by excision and in-gel trypsinization of respective spots from silver-stained gels as described previously [10]. The analyses were performed using a 4700 MALDI-TOF/TOF Proteomics Analyzer (Applied Biosystems, Foster City, CA, USA) equipped with a $355 \mathrm{~nm} \mathrm{Nd:YAG} \mathrm{laser.} \mathrm{Combined} \mathrm{MS} \mathrm{and} \mathrm{MS/}$ MS spectra were submitted to MASCOT version 2.1 (Matrix Science, London, UK) using GPS Explorer software version 3.6 (Applied Biosystems). MASCOT protein scores (based on combined MS and MS/MS spectra) greater than 72 were considered statistically significant $(\mathrm{p} \leq 0.05)$. The individual MS/MS spectrum with statistically significant (confidence interval > 95\%) best ion score (based on MS/MS spectra) were accepted. The number of identified peptides, sequence coverage and number of MS/MS identified peptides were considered during protein identification. To eliminate the redundancy of proteins that appeared in the database under different names and accession numbers, the single-protein member belonging to the species Mycobacterium with the highest protein score (top rank) was singled out from the multi-protein family [34].

\section{Expression and purification of recombinant proteins and antisera production}

The coding sequence for each protein was amplified from M. tuberculosis strain H37Rv genomic DNA by PCR. The specific primers included sequences for appropriate restriction sites to enable cloning. The primers and parameters for thermal cycler amplification are shown in Table 3. PCR products were cloned into pET28a or pET30a vectors (EMD Biosciences, San Diego, CA, USA), resulting in the addition of a $6 \times$ His $\mathrm{N}$-terminal leader sequence to each coding sequence. The fidelity of the recombinant plasmid was confirmed by sequencing. Plasmids encoding respective fusion proteins were transformed into Escherichia coli BL21 (DE3). Cell cultures were grown to $\mathrm{OD}_{600}$ 0.6-0.8 in Luria Bertani broth containing $100 \mu \mathrm{g} / \mathrm{mL}$ kanamycin. Protein induction was then carried out by adding $1 \mathrm{mM}$ isopropyl-D-thiogalactoside for 5 hours at $37^{\circ} \mathrm{C}$. Induced cells were harvested and washed with cold $50 \mathrm{mM}$ sodium phosphate buffer $(\mathrm{pH}$ 7.9) and centrifuged at $8000 \times \mathrm{g}$ for $15 \mathrm{~min}$. The cell pellet was resuspended in Ni-NTA lysis buffer $(300 \mathrm{mM}$ $\mathrm{NaCl}, 50 \mathrm{mM}$ sodium phosphate buffer, $\mathrm{pH}$ 7.9) at $50 \mathrm{~g} /$ $\mathrm{mL}$ wet weight. Bacteria were lysed by sonication and the lysate was centrifuged at $12000 \times \mathrm{g}$ for $30 \mathrm{~min}$. Supernatants were then loaded onto a Ni-NTA His-Binding column (Novagen, Madison, WI, USA) and His-tagged proteins were eluted using elution buffer containing various imidazole concentrations. Pellets were dissolved in lysis buffer in the presence of $8 \mathrm{M}$ urea and then purified using a Ni-NTA His-Binding column as described above. The purified proteins were dialyzed against $5 \mathrm{mM}$ Tris$\mathrm{HCl}$ buffer (pH 7.5) containing $100 \mathrm{mM} \mathrm{NaCl}$ and 3\% (v/ v) glycerol and quantified using Bradford reagent.

Procedures for antiserum production were based on standard protocols [37]. Briefly, 3-month-old New Zealand White rabbits were immunized with recombinant proteins emulsified with incomplete Freund's adjuvant and boosted four times at 2-week intervals, and antisera collection was performed 7 days after the last boost.

\section{Enzyme-linked immunosorbent assay}

Enzyme-linked immunosorbent assays (ELISA) were used to assess the humoral response in humans to the recombinant proteins identified in this study. Ninetysix-well microtitre plates (Nalge Nunc International, Rochester, NY, USA) were coated with $500 \mathrm{ng}$ of recombinant protein antigen dissolved in $100 \mu \mathrm{L}$ of $0.05 \mathrm{M}$ carbonic acid buffer, and incubated overnight at $4^{\circ} \mathrm{C}$ in a humidified chamber. Wells were washed three times 
Table 3 Primers sets for genes amplified by PCR

\begin{tabular}{|c|c|c|}
\hline Gene & Primer sequences $\left(5^{\prime}-3^{\prime}\right)$ & Restriction site used \\
\hline \multirow[t]{2}{*}{$R \vee 1310$} & 5' CGAATTCATGACTACCACTGCCGAAAAG 3' & Ecorl \\
\hline & 5' TATAAGCTITCACAGCTTGGCGCCGAGAC 3' & HinD III \\
\hline \multirow[t]{2}{*}{ Rv3028c } & 5' TAGGATCCATGGCTGAAGTACTGGTGCTC 3' & BamHI \\
\hline & 5' TATAAGCTTCTAGCCCTTGCGGGCCTTG 3' & HinD III \\
\hline \multirow[t]{2}{*}{$R \vee 3040$} & 5' TAGGATCCATGAATTCACCTCGCGAGCC 3' & BamHI \\
\hline & 5' TATAAGCTTCACAGCGGCCACCCGGTC 3' & HinD III \\
\hline \multirow[t]{2}{*}{$R v 2145 c$} & 5' TAGGATCCATGCCGCTTACACCTGCCG 3' & BamHI \\
\hline & 5' TAGAGCTCCTAGTIITTGCCCCGGTTGAAT 3' & Sacl \\
\hline \multirow[t]{2}{*}{ Rv0639 } & 5' TAGGATCCGTGACTACCTTCGACGGTG 3' & BamHI \\
\hline & 5' GACAAGCTTCTAGATCTTGGAGACTTGGCC 3' & HinD III \\
\hline \multirow[t]{2}{*}{ Rv3692 } & 5' TAGGATCCGTGACACAGTCCGCGTCCAAC 3' & BamHI \\
\hline & 5' TGTAAGCTTCTAGCGGGGCACCGGAACC 3' & $\operatorname{HinD}$ III \\
\hline \multirow[t]{2}{*}{ Rv0444C } & 5' TGGATCCATGACTGAACATACCGATTTTGAG 3' & BamHI \\
\hline & 5' TATAAGCTITCACCCGAGCGGCAGCTCGGC 3' & $\operatorname{HinD}$ III \\
\hline \multirow[t]{2}{*}{ Rv2031c } & 5' TAGGATCCATGGCCACCACCCTTCCCGT 3' & BamHI \\
\hline & 5' GCGAAGCTTTCAGTTGGTGGACCGGATCTG 3' & HinD III \\
\hline \multirow[t]{2}{*}{ Rv0859c } & 5' ATGGATCCATGTCCGAAGAAGCCTTCATCTAC 3' & BamHI \\
\hline & 5' TATAAGCTITTAAACCCTCTCGATGATCGTCGC 3' & HinD III \\
\hline
\end{tabular}

with PBST, blocked with $100 \mu \mathrm{L}$ of blocking buffer $(2 \%$ bovine serum albumin (w/v) in PBS) for 2 hours at $37^{\circ} \mathrm{C}$, and then washed three times with PBST. Sera corresponding to the different clinical groups were diluted 1:400 in blocking buffer and $100 \mu \mathrm{L}$ was added to each antigen-coated well. Following incubation for 1 hour at $37^{\circ} \mathrm{C}$, the plates were washed five times with PBST and then incubated with horseradish peroxidase-conjugated goat anti-human IgG at $37^{\circ} \mathrm{C}$ for 1 hour. Antibody binding was detected using $o$-phenylenediamine tetrahydrochloride (Sigma Aldrich, Saint Louis, MO, USA) dissolved $(1 \mu \mathrm{L} / \mathrm{mL})$ in citrate-phosphate buffer ( $\mathrm{pH} 5.4$ ) and $\mathrm{H}_{2} \mathrm{O}_{2}$. The reaction was stopped by the addition of $50 \mu \mathrm{L}$ of $0.5 \mathrm{M} \mathrm{H}_{2} \mathrm{SO}_{4}$, and absorbance values were measured at $492 \mathrm{~nm}$ in an ELISA reader (Bio-Rad). Experiments were repeated at least twice with similar results.

The ELISA results were analyzed using cut-off values equal to the mean optical density for the healthy control serum samples plus two standard deviations. Any sample exhibiting an absorbance value above the cut-off value was considered to be positive. For statistical analysis, the differences between groups of TB patients and healthy controls were calculated by the independentsamples $t$-test using the Statistics Package for Social Science (SPSS 13.0, Chicago, IL, USA), with $\mathrm{p}<0.05$ considered to be statistically significant.

\section{Additional material}

Additional file 1: PMF and MS MS Spectra of Rv0444c.

Additional file 2: PMF and MS MS Spectra of Rv0639.

Additional file 3: PMF and MS MS Spectra of Rv1310.
Additional file 4: PMF and MS MS Spectra of Rv2031c.

Additional file 5: PMF and MS MS Spectra of Rv2145c.

Additional file 6: PMF and MS MS Spectra of Rv3028c.

Additional file 7: PMF and MS MS Spectra of Rv3040.

Additional file 8: PMF and MS MS Spectra of Rv3692.

\section{Acknowledgements}

This work was supported by a grant from the 11th Five Years Programs for prevention and cure of great infectious diseases (No. 2009ZX10004-313, No. 2012ZX10003-002), and International collaboration on drug and diagnostics innovation of tropical diseases in PR China (No.2010DFB73280). The authors are grateful to Ms. ChunYu LiJuan, Mr. Xu YunMin(FuDan University) for technical support, Dr. Jin RuiLiang(Shanghai Pulmonary Hospital) for preparation of M. tuberculosis clinical strains, Ms. Yan Jituan for the collection of serum.

\section{Author details}

${ }^{1}$ State Key Laboratory of Genetic Engineering, Institute of Genetics, School of Life Science, Fudan University, 220 Handan Road, 200433 Shanghai, Peoples Republic of China. ${ }^{2}$ Shanghai Centre for Clinical Laboratory, 200126 Shanghai, Peoples Republic of China. ${ }^{3}$ Shanghai Pulmonary Hospital, Tongji University, 200433 Shanghai, Peoples Republic of China.

\section{Authors' contributions}

ZL conceived of the idea for the immunoproteomic study, participated in its design, performed a major portion of the data analysis, and drafted the manuscript; WQZ carried out immunoproteomic analysis and contributed in the preparation of the manuscript; WWJ carried out protein preparation, $2 \mathrm{DE}$, and western blot analysis; LYY assisted in protein sample extraction and western blot analysis; WJ contributed to the bioinformatics analysis; XY carried out ELISA; XWX and CZL participated in the design, implementation, and coordination of the study; $Z X L$ and $W H H$ reviewed the design plan and participated in revision of the final version of the manuscript. All authors read and approved the final manuscript.

\section{Competing interests}

The authors declare that they have no competing interests. 
Received: 27 August 2011 Accepted: 25 February 2012

Published: 25 February 2012

\section{References}

1. WHO: Guidelines for surveillance of drug resistance in tuberculosis (document WHO/HTM/TB/2009.422). Geneva: World Health Organization; 2009.

2. WHO: Anti-tuberculosis drug resistance in the world. Third global report. The WHO/IUATLD Global Project on Anti-Tuberculosis Drug Resistance Surveillance(WHO/CDC/TB/2004). Geneva: World Health Organization; 2004

3. Dorman SE, Chaisson RE: From magic bullets back to the Magic Mountain: the rise of extensively drug-resistant tuberculosis. Nat Med 2007, 13:295-298.

4. Agranoff D, Fernandez-Reyes D, Papadopoulos MC, Rojas SA, Herbster M, Loosemore A, Tarelli E, Sheldon J, Schwenk A, Pollok R, Rayner CFJ, Krishna S: Identification of diagnostic markers for tuberculosis by proteomic fingerprinting of serum. Lancet 2006, 368:1012-1021.

5. Marris E: From TB tests, just a 'yes or no' answer, please. Nat Med 2007, 13:267.

6. Lyashchenko KR, Colangele M, Houde HA, Jahadali D, Menizies GML: Heterogenous antibody responses in tuberculosis. Infect Immun 1998, 66:3936-3940.

7. Abebe F, Holm-Hansen C, Wiker HG, Bjune G: Progress in Serodiagnosis of Mycobacterium tuberculosisInfection. Scand J Immunol 2007, 66:176-191.

8. Starck J, Kallenius G, Marklund Bl, Andersson DI, Akerlund T: Comparative proteome analysis of Mycobacterium tuberculosi grown under aerobic and anaerobic conditions. Microbiol 2004, 50:821-3829.

9. Betts JC, Lukey PT, Robb LC, McAdam RA, Duncan K: Evaluation of a nutrient starvation model of Mycobacterium tuberculosi persistence by gene and protein expression profiling. Mol Microbiol 2002, 43:717-731.

10. Jungblut PR, Schaible UE, Mollenkopf HJ, Zimny-Arndt U, Raupach B, Mattow J, Halada P, Lamer S, Kaufmann SHE: Comparative proteome analysis of Mycobacterium tuberculosi and Mycobacterium bovi BCG strains: towards functional genomics of microbial pathogens. Mol Microbiol 1999, 33:1103-1117.

11. Mattow J, Jungblut PR, Schaible UE, Mollenkopf HJ, Lamer S, ZimnyArndt U, Hagens K, Müller EC, Kaufmann SHE: Identification of proteins from Mycobacterium tuberculosi missing in attenuated Mycobacterium bovi BCG strains. Electrophoresis 2001, 22:2936-2946.

12. Mawuenyega KG, Forst CV, Dobos KM, Belisle JT, Chen J, Bradbury EM, Bradbury ARM, Chen X: Mycobacterium tuberculosis Functional Network Analysis by Global Subcellular Protein Profiling. Mol Biol Cell 2005 16:396-404.

13. Mattow J, Schaible UE, Schmidt F, Hagens K, Siejak F, Brestrich G, Haeselbarth G, Müller EC, Jungblut PR, Kaufmann SHE: Comparative proteome analysis of culture supernatant proteins from virulent Mycobacterium tuberculosis $\mathrm{H} 37 \mathrm{Rv}$ and attenuated $M$-bovi BCG Copenhagen. Electrophoresis 2003, 24:3405-3420.

14. Pheiffer C, Betts JC, Flynn HR, Lukey PT, van Helden P: Protein expression by a Beijing strain differs from that of another clinical isolate and Mycobacterium tuberculosis H37Rv. Microbiology 2005, 151:1139-1150.

15. Mattow J, Siejak F, Hagens K, Becher D, Albrecht D, Krah A, Schmidt F, Jungblut PR, Kaufmann SHE, Schaible UE: Proteins unique to intraphagosomally grown Mycobacterium tuberculosis. Proteomics 2006, 6:2485-2494

16. Sanchez-Campillo M, Bini L, Comanducci M, Raggiaschi R, Marzocchi B, Pallini V, Ratti G: Identification of immunoreactive proteins of Chlamydia trachomatis by Western blot analysis of a two-dimensional electrophoresis map with patient sera. Electrophoresis 1999, 20:2269-2279.

17. Jungblut PR: Proteome analysis of bacterial pathogens. Microbes Infect 2001, 3:831-840.

18. Tjalsma H, Schaeps MJR, Swinkels WD: Immunoproteomics: From biomarker discovery to diagnostic applications. Proteomics Clin 2008, 2:167-180

19. Bassey EOE, Catty D, Kumararatne DS, Raykundalia C: Candidate antigens for improved serodiagnosis of tuberculosis. Tuber Lung Dis 1996, 77:136-145.

20. Samanich KM, Belisle JT, Sonnenberg MG, Keen MA, Zolla-Pazner S, Laal S: Delineation of Human Antibody Responses to Culture Filtrate Antigens of Mycobacterium tuberculosis. J Infect Dis 1998, 178:534-1538.
21. Charlet D, Mostowy S, Alexander D, Sit L, Wiker HG, Behr MA: Reduced expression of antigenic proteins MPB70 and MPB83 in Mycobacterium bovis BCG strains due to a start codon mutation in sigK. Mol Microbiol 2005, 56:1302-1313.

22. Saïd-Salim B, Mostowy S, Kristof AS, Behr MA: Mutations in Mycobacterium tuberculosis Rv0444c, the gene encoding anti-SigK, explain high level expression of MPB70 and MPB83 in Mycobacterium bovis. Mol Microbiol 2006, 62:1251-1263.

23. Preneta $R$, Papavinasasundaram KG, Cozzone AJ, Duclos B: Autophosphorylation of the $16 \mathrm{kDa}$ and $70 \mathrm{kDa}$ antigens (Hsp 16.3 and Hsp 70) of Mycobacterium tuberculosis. Microbiol 2004, 150:2135-2141.

24. Beck ST, Leite OM, Arruda RS, Ferreira AW: Humoral response to low molecular weight antigens of Mycobacterium tuberculosis by tuberculosis patients and contacts. Braz J Med Biol Res 2005, 38:587-596.

25. Demissie A, Leyten EM, Abebe M: Recognition of stage-specific mycobacterial antigens differentiates between acute and latent infections with Mycobacterium tuberculosis. Clin Vaccine Immunol 2006 13:179-186.

26. Kyte J, Doolittle RF: A simple method for displaying the hydropathic character of a protein. J Mol Biol 1982, 157:105-132.

27. Zhang $M$, Yang YP, Xu Y, Qie YQ, Wang JL, Zhu BD, Wang QZ, Jin RL, Xu SF, Wang HH: Trehalose-6-phosphate Phosphatase from Myco bacterium tuberculosis induces humoral and cellular immuneresponses. FEMS Immunol Med Microbiol 2007, 49:68-74.

28. Wallis RS, Pai M, Menzies D, Doherty TM, Walzl G, Perkins MD, Zumla A: Biomarkers and diagnostics for tuberculosis: progress, needs, and translation into practice. Lancet 2010, 75(9729):1920-1937.

29. Shreemanta KP, Kaufmann SHE: The quest for biomarkers in tuberculosis. Drug Discov Today 2010, 15(3/4):148-157.

30. Pedersen SK, Sloane AJ, Prasad SS, Sebastian LT, Lindner RA, Hsu M, Robinson M, Bye PT, Weinberger RP, Harry JL: An Immunoproteomic approach for identification of clinical biomarkers for monitoring disease. Mol Cell Proteomics 2005, 4:1052-1060

31. Leroy B, Roupie V, Noel-Georis I, Rosseels V, Walravens K, Govaerts M, Huygen K, Wattiez R: Antigen discovery, A postgenomic approach to paratuberculosis diagnosis. Proteomics 2007, 7:1164-1176.

32. Ramaswamy SV, Reich R, Dou SJ, Jasperse L, Pan X, Wanger A, Quitugua T, Graviss EA: Single nucleotide polymorphisms in genes associated with isoniazid resistance in Mycobacterium tuberculosis. Antimicrob Agents Ch 2003, 47:1241-1250.

33. Bahk YY, Kim SA, Kim JS, Euh YJ, Bai GH, Cho SN, Kim YS: Antigens secreted from Mycobacterium tuberculosis: identification by proteomics approach and test for diagnostic marker. Proteomics 2004, 4:1-9.

34. Zheng XJ, Hong LL, Shi LX, Guo JQ, Sun Z, Zhou JY: Proteomic analysis of host cells infected with infectious bursal disease virus. Mol Cell Proteomics 2008, 7:612-625.

35. Gharahdaghi F, Weinberg CR, Meagher DA, Imai BS, Mische SM: Mass spectrometric identification of proteins from silver-stained polyacrylamide gel: A method for the removal of silver ions to enhance sensitivity. Electrophoresis 1999, 20:601-605.

36. Griffin TJ, Gygi SP, Ideker T, Rist B, Eng J, Hood L, Aebersold R: Complementary profiling of gene expression at the transcriptome and proteome levels in Saccharomyces cerevisiae. Mol Cell Proteomics 2002, 1:323-333.

37. Sambrook J, Fritsch EF, Maniatis T: Molecular Coning: A Laboratory Manual. 2 edition. New York: Cold Spring Harbor Laboratory Press; 1989.

doi:10.1186/1477-5956-10-12

Cite this article as: Zhang et al:: Identification of putative biomarkers for the serodiagnosis of drug-resistant Mycobacterium tuberculosis. Proteome Science 2012 10:12. 